\title{
Cavitation Limits on Tidal Turbine Performance
}

\author{
Aidan Wimshurst, Christopher Vogel, Richard Willden \\ Department of Engineering Science, University of Oxford, Parks Road, OX1 3PJ, UK
}

\begin{abstract}
Blockage effects are currently not accounted for in cavitation analyses of tidal turbine rotors. At higher blockage ratios, rotors are more heavily loaded and have potentially stronger suction peaks, so cavitation inception is more likely. In this paper, blade resolved computations are used to carry out a cavitation analysis over a range of blockage ratios and tip speed ratios. Our analysis suggests that increasing the blockage ratio from 0.01 to 0.197 reduces the minimum static pressure head in the fluid by approximately $0.5 \mathrm{~m}$. To mitigate this reduction, either the submersion depth of the rotor can be increased or the maximum permissible tip speed ratio reduced. However, reducing the maximum permissible tip speed ratio is shown to severely restrict the rotor thrust and power. Spanwise flow effects are shown to reduce the strength of the suction peak on the outboard blade sections, reducing the likelihood of cavitation inception. Blade element based methods are shown to inadequately account for spanwise flow effects and thus are overly conservative. Hence, rotors designed with these methods could potentially be operated at higher tip speed ratios or reduced submersion depths.
\end{abstract}

Keywords: Cavitation, Blockage, Spanwise Flow, Safety Margin, Tidal Turbines

\section{Introduction}

There is a general desire to increase the design tip-speed-ratio of tidal turbine rotors, which is often around 4-6 and is much lower than the design tip-speed-ratio of utility-scale wind turbines (7-11). Higher design tip-speed-ratios increase the maximum theoretical power coefficient that can be achieved by the rotor 5 and also reduce the maximum torque load, so that a lighter drive train can be adopted with a reduced structural cost (Ning and Dykes, 2014). However, unlike onshore wind turbines (where the tip-speed-ratio is limited by noise constraints), the tip-speed-ratio of tidal turbine rotors is limited by the onset of cavitation.

Bahaj et al. (2007) and Wang et al. (2007) observed the onset of tip vortex cavitation in cavitation tunnels downstream of their experimental tidal turbine rotors. Reducing the static pressure in the tunnel led to 10 the inception of back sheet cavitation on the suction surface of the rotor blade and increased the strength of the tip vortex cavitation. While these experiments are useful for classifying the type of cavitation and observing its onset, tidal turbines need to be designed to avoid the onset of cavitation altogether (DNV GL, 2015). The majority of tidal turbine rotor design algorithms that have been adopted are based on the Blade Element (BE) method, where the rotor blade is divided into several independent aerofoil sections along the span. The forces acting on each aerofoil section are assumed to arise only from the flow components in the plane of the aerofoil section. In the Blade Element Momentum (BEM) method, the BE method is combined with linear and angular momentum conservation equations for each annulus of the rotor disc, to compute the spanwise variation of the angle of attack and relative velocity magnitude (Burton et al., 2011). Batten et al. (2008) used this method to design a tidal turbine rotor, with a cavitation bucket type analysis (originally used in propeller design) to assess the onset of cavitation. This algorithm has subsequently been adopted

\footnotetext{
* Corresponding author

Email address: Aidan.Wimshurst@eng.ox.ac.uk (Aidan Wimshurst)
} 
by other authors to design tidal turbine rotors, including Goundar and Ahmed (2013) and Grogan et al. (2013).

However, algorithms based on the BEM method are only able to design rotors for optimum operation in unblocked conditions (where the device is operating in relative isolation from flow boundaries and other devices). In practice, tidal turbine rotors are likely to be grouped closely together in arrays, allowing them to utilise the increased blockage provided by the free surface, sea bed and other neighbouring devices (Adcock et al., 2015; Vennel et al., 2015). The increased blockage leads to a greater static pressure drop in the streamwise direction across the device (than occurs in unblocked conditions), so the device can support greater thrust and extract more power from the flow. Under these conditions, optimum rotors need to be designed to apply greater thrust to the flow than in unblocked conditions, so that they can extract the increased power that is available (Schluntz and Willden, 2015). McIntosh et al. (2011) proposed an algorithm to design tidal turbine rotors specifically for operation under blocked conditions. Rather than combining the BE method with algebraic momentum equations, they embedded the BE method within a 3D RANS solver, which can account for blockage directly through the boundary conditions of the domain. When operated at

35 the blockage ratio they were specifically designed for, rotors that were designed for blockage have been shown to universally outperform rotors that were not designed specifically for that blockage condition (Schluntz and Willden, 2015).

Despite the adoption of these BE based design algorithms, the effect of blockage on cavitation inception has not been examined directly. With increasing blockage ratio, rotors are more heavily loaded, can produce

40 higher power and have potentially stronger suction peaks. Hence, rotors are more likely to cavitate at higher blockage ratios, since the minimum static pressure in the fluid is reduced. Furthermore, blade resolved computations performed by Wimshurst and Willden (2017) have observed significant spanwise flow (40-50\% of the freestream velocity) on the outboard sections of tidal turbine rotor blades. This spanwise flow changes the surface pressure distribution on the outboard blade sections and reduces the strength of

45 the suction peak. As the magnitude of the suction peak reduces, the minimum static pressure in the fluid increases, so the fluid is less likely to cavitate. Hence, BE based design algorithms that do not adequately account for spanwise flow effects are likely to inaccurately predict cavitation inception.

In this work, a cavitation analysis will be carried out using a series of blade resolved computations, which account for blockage and spanwise flow effects directly. The results of these computations will be 50 compared with the results of a separate cavitation analysis carried out with a BE based method, to quantify the discrepancies in the BE method. In addition, a hydrodynamic safety margin will be proposed and included in the analysis to account for ambient turbulence, free surface waves and velocity shear (which are not modelled explicitly). This safety margin increases the minimum allowable static pressure on the suction surface of the blade, to account for the unsteady pressure fluctuations which have not been modelled. It can be realised in practice by either reducing the maximum permissible tip-speed-ratio of the rotor or increasing the depth of submersion. To complete the analysis, the resulting restriction on the range of thrust and power coefficients that can be achieved by the rotor will be investigated, when the maximum permissible tip-speed-ratio is limited in order to avoid the onset of cavitation.

\section{Tidal Turbine Rotor Designs}

Two different tidal turbine rotors are investigated in this work. These rotors were derived from the original designs of Schluntz and Willden (2015) by Wimshurst and Willden (2016) and will be referred to as 'Rotor 1' and 'Rotor 2' respectively. Rotor 1 was designed to operate in a virtually unblocked domain (a blockage ratio of 0.0001), while Rotor 2 was designed to operate in a more highly blocked domain (a blockage ratio of 0.196$)$. Here the 'blockage ratio' $(B)$ refers to the ratio of the swept area of the rotor to the cross-sectional area of the computational domain that the device resides in. Both rotors were designed to achieve their maximum power coefficients at a tip-speed-ratio of 5 (the design tip-speed-ratio), in the domains they were originally designed for. In this work, the tip-speed-ratio $\lambda$ is defined as $\lambda=\Omega R / U_{\infty}$, where $\Omega$ is the rotational speed of the rotor, $R$ is the rotor radius and $U_{\infty}$ is the freestream velocity (which is held constant at $2.0 \mathrm{~m} / \mathrm{s}$ ). 
Fig. 1 shows the chord $(c)$ and twist $(\beta)$ distributions for Rotor 1 and Rotor 2 . Both rotors use the same RIS $\varnothing-A 1-24$ aerofoil along the entire span and have a total radius $(R)$ of $10 \mathrm{~m}$. The main difference between the rotor designs is that Rotor 2 has a greater local chord length and a slightly lower twist angle along the entire span. This allows it to apply greater thrust to the flow when operating at the same tip-speed-ratio as Rotor 1 .
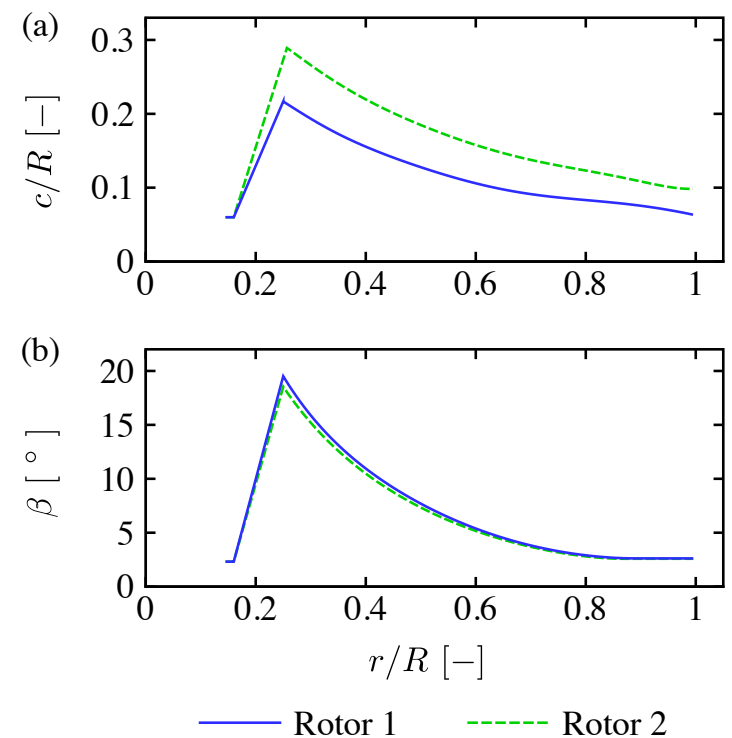

Figure 1: Chord (a) and twist (b) distributions for Rotor 1 and Rotor 2. The rotor radius $R=10 \mathrm{~m}$. The RIS $\varnothing$-A1-24 aerofoil is blended into a cylinder from $0.175 \leq r / R \leq 0.25$.

Inboard of $r / R=0.25$, the RIS $\varnothing-A 1-24$ aerofoil was blended into a cylinder to facilitate pitching at the blade root. A cylindrical nacelle with diameter $0.15 D$ (where $D$ is the rotor diameter), length $0.5 D$ and a hemispherical nose cone, were also included in both rotor designs. These dimensions were chosen based on observations of some full-scale tidal turbine demonstrator devices that have already been installed (Belloni, 2013).

When analysed in a series of blade resolved computations by Wimshurst and Willden (2016), both rotors were found to achieve their maximum power coefficients close to the original design tip-speed-ratio of 5.0, in the domains they were originally designed for. More specifically, Rotor 1 achieved its maximum power coefficient in a low blockage domain (a blockage ratio of 0.01) at a tip-speed-ratio of 5.27, while Rotor 2 achieved its maximum power coefficient in a more highly blocked domain (a blockage ratio of 0.197 ) at a tip-speed-ratio of 5.18 .

The blade resolved computations of Wimshurst and Willden (2016) will also be used for the cavitation analysis in this work. However, before presenting this analysis, some additional details of the blade resolved computations will be provided in the next section.

\section{Blade Resolved Computations}

The blade resolved computations were carried out in steady uniform flow, using the Multiple Reference Frame (MRF) approach of Luo et al. (1994) to simulate rotor rotation, over a range of blockage ratios (0.01, $0.065,0.0982$ and 0.197$)$ and tip-speed-ratios $(4-7)$. A freestream velocity $\left(U_{\infty}\right)$ of $2.0 \mathrm{~m} / \mathrm{s}$ was adopted for all simulations and the rotational speed of the rotor was varied to set the tip-speed-ratio. To reduce the computational cost of each simulation, only a third of the rotor (a single blade and a section of the nacelle) was simulated in each computation. As shown in Fig. 2, the computational domain took the form of a $120^{\circ}$ wedge shape, with periodic boundary conditions applied on the sides of the domain. The radius of the outer domain was varied to set the blockage ratio. The inlet turbulence intensity was set to $10.0 \%$, with a 
turbulence length scale of $14 \mathrm{~m}$, to mimic the high levels of turbulence found in real tidal channels, following Gant and Stallard (2008).

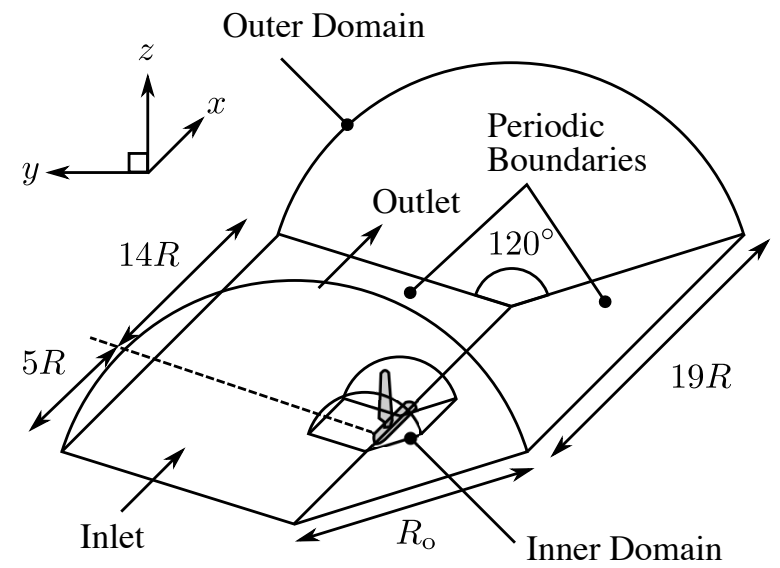

Figure 2: Schematic diagram of the computational domain adopted in the blade resolved computations. The streamwise flow direction is in the positive $x$ direction and the rotor rotates clockwise as viewed from the front. The radius of the outer domain $\left(R_{\mathrm{o}}\right)$ is varied to set the blockage ratio.

OpenFOAM (version 2.3.1) was used to solve the governing Reynolds-averaged Navier-Stokes (RANS) equations, with the $k-\omega$ SST turbulence model proposed by Menter (1994) for turbulence closure. Spatial convergence was assessed by refining the block structured mesh twice and then comparing the forces on a blade section at $95 \%$ of the blade span (between the three levels of refinement). With the coarsest mesh ( 2.1 million cells), the axial force per unit span (the thrust producing force) was within $0.37 \%$ of the finest mesh, while the tangential force per unit span was within $0.63 \%$ of the finest mesh (5.5 million cells). Hence, the coarsest mesh was deemed to be sufficiently accurate to compute the surface pressure distribution on the outboard blade sections. This mesh utilised 68 cells in the spanwise direction, 58 cells in the chordwise direction (along the pressure and suction surfaces individually) and a wall function approach was adopted to model the boundary layer, with a growth ratio of 1.1 normal to the wall and a wall normal distance in the range of $30<y^{+}<200$ over the entire surface of the blade.
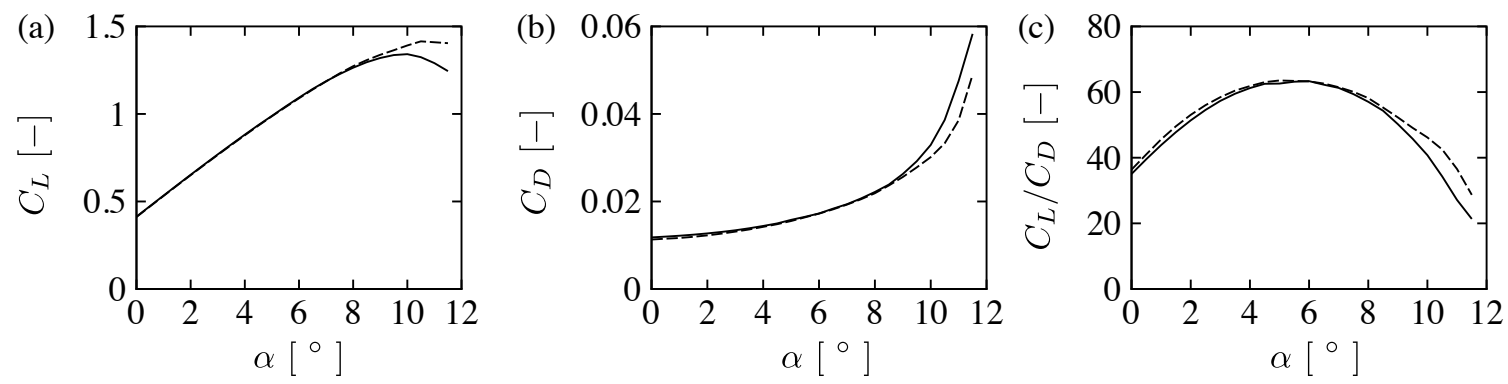

- Viscous Sub-Layer Resolved

Wall Functions

Figure 3: Computed sectional (a) lift coefficient, (b) drag coefficient and (c) lift to drag ratio for the RIS $\varnothing$-A1-24 aerofoil at a chord based Reynolds number of 12 million, with wall function modelling and viscous sub-layer resolution.

To investigate the limitations of the wall function approach, a comparison was made of the aerodynamic performance of the RIS $\varnothing-\mathrm{A} 1-24$ aerofoil (in 2D) with viscous-sub layer resolved $\left(y^{+}<5\right)$ and wall function $\left(30<y^{+}<200\right)$ based meshes. The mesh structure for the 2D meshes was specifically constructed to closely resemble the mesh structure on a slice through the $3 \mathrm{D}$ rotor blade (normal to the blade axis) at $r / R=0.95$. The computations were carried out over a range of angles of attack from $0^{\circ}$ to $11.5^{\circ}$, in increments of $0.5^{\circ}$ at 
a chord based Reynolds number of 12 million. A chord based Reynolds number of 12 million (approximately) corresponds with the chord based Reynolds number experienced by an aerofoil section at $r / R=0.95$ where the chord is $1.25 \mathrm{~m}$, the kinematic viscosity is $1 \times 10^{-6} \mathrm{~m}^{2} / \mathrm{s}$ and the incident velocity (accounting for rotation but ignoring induction) is $9.5 \mathrm{~m} / \mathrm{s}$ at a tip-speed-ratio of 5 . Fig. 3 shows the sectional lift coefficient $\left(C_{L}\right)$, sectional drag coefficient $\left(C_{D}\right)$ and sectional lift to drag ratio $\left(C_{L} / C_{D}\right)$, for the viscous sub-layer resolved and wall function based meshes. For angles of attack below $8^{\circ}$, the lift and drag coefficients are both well predicted by the wall function based mesh. As a result, the peak lift to drag ratio (at approximately $6^{\circ}$ ) is also well predicted and hence the 3D rotor mesh is expected to predict the peak power coefficient well at the design tip-speed-ratio. However, the wall function based mesh over-predicts the lift coefficient and under-predicts the drag coefficient at high angles of attack (above $8^{\circ}$ ), due to a delayed separation onset by the wall function based mesh. However, this is not likely to significantly impact the results of this investigation, since cavitation inception is more likely to occur at high tip-speed-ratios and thus low angles of attack (which are well predicted by the wall function based mesh).

\section{Blade Element Computations}

The Blade Element (BE) model adopted for these computations is a steady flow RANS-embedded Blade Element Actuator Disk (BE-AD) model, rather than a purely analytical BEM model. This embedded BE model was chosen to minimise the modelling differences between the blade resolved and BE approaches, in order to isolate the differences caused by three-dimensional flow effects. More specifically, the rotor, nacelle, and domain boundaries for the BE-AD simulations matched the blade resolved simulations exactly (with the exception that the full $360^{\circ} B=0.01$ domain was resolved, rather than a $120^{\circ}$ segment). Hence, the flow deflection by the relatively large nacelle ( $15 \%$ of the rotor diameter) could be captured in the BE-AD approach.

Many of the computations were carried out at high tip-speed-ratios, as cavitation inception is more likely to occur here. At high tip-speed-ratios, the analytical BEM method requires an empirical correction to account for the turbulent wake state that arises under high thrust conditions. While several different empirical corrections have been proposed and calibrated to wind turbine rotors under high induction conditions (such as the Glauert (1935) correction), the accuracy of these corrections remains uncertain when applied to high solidity tidal turbine rotors. However, the BE-AD method does not require an empirical correction under these conditions, since the axial induction at the rotor plane is computed directly from the RANS equations.

As with most $\mathrm{BE}$ based models, the BE-AD model adjusts for the finite number of blades in the azimuthally-averaged analysis by modifying the axial induction factor with the Glauert tip correction factor (Glauert, 1935). The increase in the axial induction factor on the outboard blade sections reduces the implied angle of attack, which consequently reduces the strength of the blade suction peak. Hence, the Glauert correction does (indirectly) lead to a reduction in strength of the suction peak on the outboard blade sections. However, the Glauert correction factor is only an approximation to the behaviour of real rotors and hence spanwise flow effects are not necessarily predicted with sufficient accuracy when using this approach. The resulting discrepancy between the blade resolved computations and the BE-AD approach will be demonstrated and discussed in more detail in Section 6 .

The BE-AD computations were carried out using an in-house code developed at the University of Oxford (McIntosh et al., 2011), implemented as a set of user defined functions in ANSYS Fluent 15.0, which was used to solve the RANS equations. The turbine rotor was modelled as a porous actuator disc, with the axial (thrust producing) and tangential (torque producing) blade forces computed as a function of the simulated flow speed through the actuator disc. The resultant static pressure drop across the disc and increase in swirl velocity were imposed on the flow by the actuator disc, and the RANS equations solved iteratively until convergence was achieved. CFD computed aerofoil data (from Fig. 3) was used instead of experimental aerofoil data, in order to eliminate a potential source of discrepancy between the BE-AD computations and the blade resolved analyses.

For the BE-AD computations, spatial mesh convergence was assessed by monitoring the azimuthallyaveraged axial and tangential blade forces at $95 \%$ of the blade span with three levels of mesh refinement. The coarsest mesh, with 1.2 million cells, yielded forces per unit span within $0.5 \%$ of that of the finest mesh 
(3.8 million cells), and hence the coarsest mesh was considered to be sufficiently accurate for the comparison with the results of the blade resolved computations in the low blockage domain $(B=0.01)$ in Section 6 .

\section{Cavitation Analysis}

The precise onset of cavitation is difficult to predict exactly as it depends on the local static pressure, the amount of dissolved air in the fluid and the time taken for the cavitation nuclei to grow to a sufficient size (Carlton, 1994). For simplicity, cavitation inception is often assumed to occur when the minimum static pressure in the fluid drops below the vapour pressure of the fluid. This simplified approach will be used in the current analysis. This was deemed a reasonable approximation, since the aim of this work is to avoid the onset of cavitation altogether, rather than modelling the cavitation process itself.

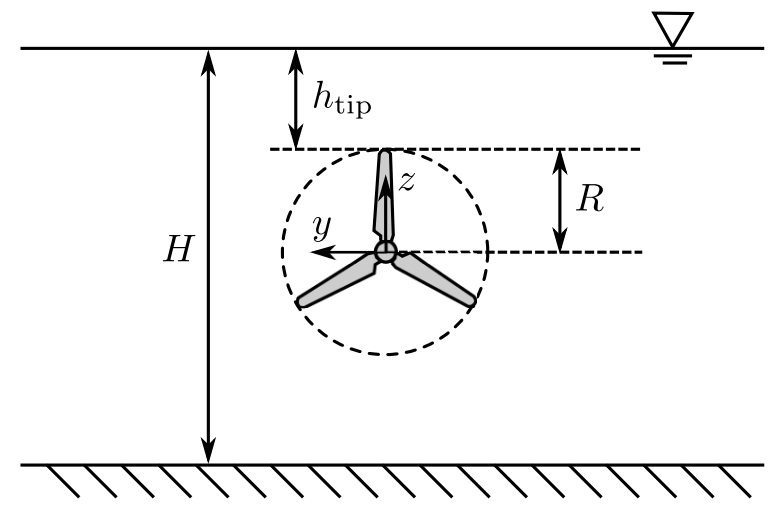

Figure 4: Schematic diagram of the domain adopted for the cavitation analysis. The streamwise $(x)$ direction is into the page. $H$ is the depth of the fluid.

However, the static pressure distribution extracted from the results of the blade resolved computations cannot be compared directly with the vapour pressure of seawater to assess the likelihood of cavitation inception. This is because a rigid lid model was adopted for the free surface of the blade resolved computations, so the hydrostatic and atmospheric pressure components were not included in the analysis. The rigid lid model also neglects the drop in free surface height that must accompany energy extraction. However, the rigid lid model has been shown in the literature to be sufficiently accurate under realistic operating conditions and was therefore also deemed to be acceptable for this investigation. Consul et al. (2013) for example, showed that even under high thrust conditions and a (relatively high) blockage ratio of 0.4 , the maximum free surface deformation in the vicinity of their tidal energy device was only $0.5 \%$ of the undisturbed fluid depth.

In this investigation, the hydrostatic and atmospheric pressure components therefore need to be added during the post-processing stage, so that a cavitation analysis can be carried out. To add hydrostatic and atmospheric components to the results of the blade resolved computations, Fig. 4 shows a schematic diagram of the assumed location of the rotor within a tidal channel. As the hydrostatic pressure is a minimum closest to the free surface (and hence cavitation inception is most likely to occur here), the blade is considered to be located at top dead centre. In addition, a tip clearance $\left(h_{\text {tip }}\right)$ of $8 \mathrm{~m}$ was adopted for the blade, based on the minimum tip submersion specified in the MeyGen Project (MeyGen Ltd., 2014). Using the notation and coordinate system shown in Fig. 4, the static pressure in the fluid $(p)$ can be assembled from the computed $\left(p_{\mathrm{CFD}}\right)$, atmospheric $\left(p_{\mathrm{Atm}}=101325 \mathrm{~Pa}\right)$ and hydrostatic components, where $z$ is the vertical coordinate measured upwards from the centre of the nacelle.

$$
p=p_{\mathrm{CFD}}+p_{\mathrm{Atm}}+\rho g\left(h_{\mathrm{tip}}+R-z\right)
$$

Fig. 5 shows the resulting static pressure distribution on the suction surface of the blade of Rotor 1 , at a tip-speed-ratio of 5.0. The static pressure distribution has several competing contributions. While the 


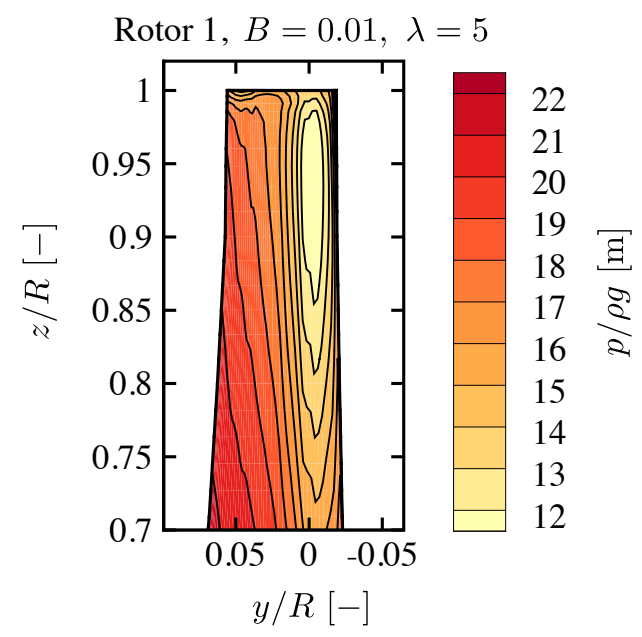

Figure 5: Contours of static pressure on the suction surface of Rotor 1 at a tip-speed-ratio of 5.0, a blockage ratio of 0.01 and a tip submersion depth of $8 \mathrm{~m}$. The streamwise $(x)$ direction is into the page and the rotor rotates clockwise.

rotational velocity component increases with distance from the hub (increasing the strength of the suction developed on the suction surface), spanwise flow effects cause the suction developed to drop off as the tip is approached. Furthermore, the hydrostatic pressure contribution reduces with distance along the blade (as the blade is located at top dead centre). Hence, the minimum static pressure does not occur at the very tip of the blade but slightly inboard from the tip, at $r / R \approx 0.94$.

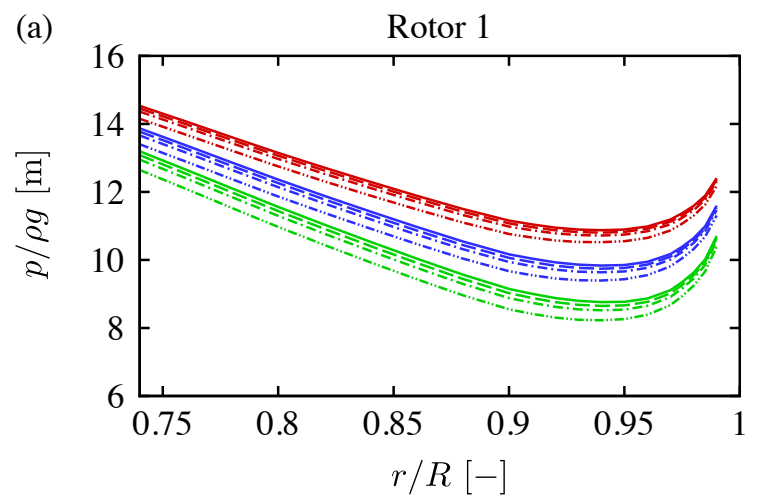

$$
\begin{aligned}
-B & =0.01, \lambda=5 \\
-\cdots & =0.065, \quad \lambda=5 \\
-\cdots & =0.0982, \quad \lambda=5 \\
\cdots \cdots & =0.197, \quad \lambda=5
\end{aligned}
$$

(b) $\quad$ Rotor 2

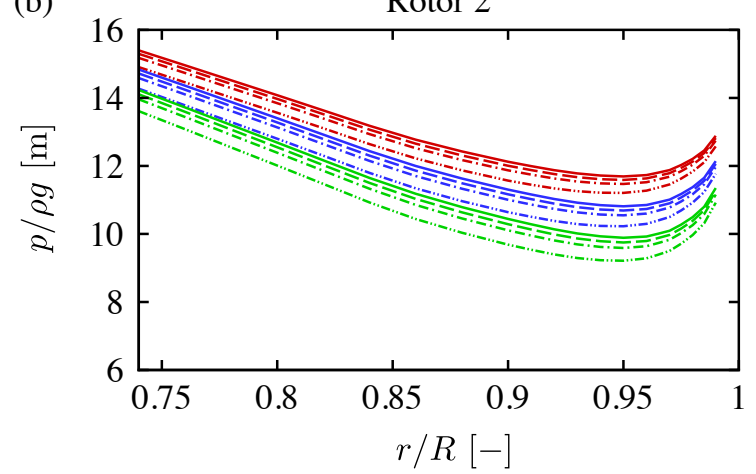

Figure 6: Spanwise variation of the minimum static pressure acting on each aerofoil section, expressed in terms of a pressure head. The tip submersion depth $h_{\text {tip }}=8 \mathrm{~m}$.

To assess the spanwise variation of the minimum static pressure in more detail, the rotor blade was divided into several independent spanwise stations. On each of these stations, the minimum static pressure was extracted (via equation 1) to give the spanwise variation of the minimum static pressure shown in Fig. 6. With increasing tip-speed-ratio, the rotational velocity component increases, increasing the dynamic pressure incident on the blade and the magnitude of the suction peak. Hence, the minimum static pressure is seen to reduce in magnitude with increasing tip-speed-ratio and the rotor is pushed closer towards flow 
cavitation.

(a)

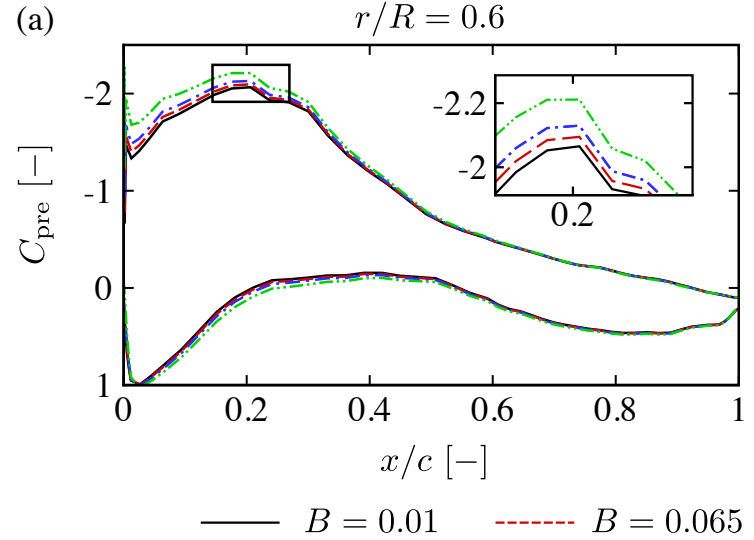

(b)

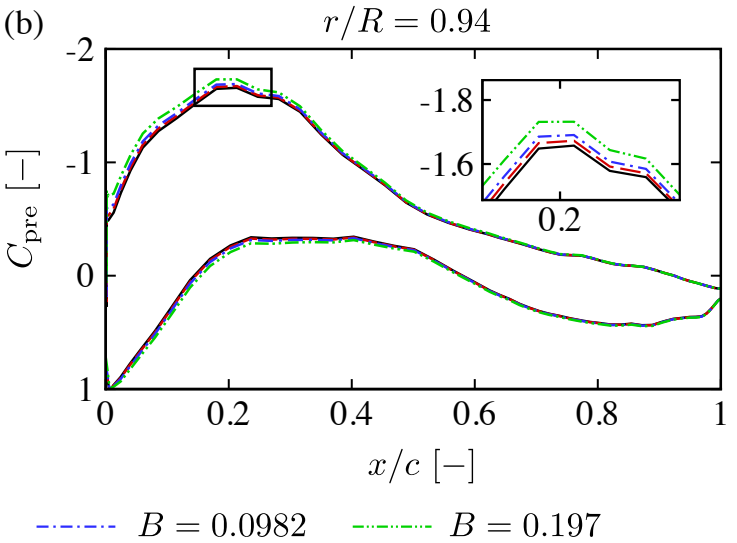

Figure 7: Surface pressure coefficient in the chordwise direction on a slice through Rotor 1 (normal to the blade axis) at (a) $r / R=0.6$ and (b) $r / R=0.94$, for a range of blockage ratios. The tip-speed-ratio $\lambda=5$.

Fig. 6 also shows that increasing the blockage ratio at constant tip-speed-ratio reduces the minimum static pressure, pushing the rotor closer towards flow cavitation. This reduction occurs because increasing the blockage ratio at a constant tip-speed-ratio increases the axial velocity through the swept area of the rotor. As the axial velocity increases, it can be inferred (by considering a velocity triangle) that the angle of attack and relative velocity magnitude increase, leading to a stronger suction peak at a given tip-speed-ratio, as shown in Fig. 7. Here the static pressure coefficient has been defined as,

$$
C_{\text {pre }}=\frac{p_{\mathrm{CFD}}-p_{\infty}}{\frac{1}{2} \rho\left[U_{\infty}^{2}+(\Omega r)^{2}\right]}
$$

where $\Omega$ is the rotational speed of the rotor, $r$ is the spanwise distance along the blade and $p_{\infty}$ is the freestream static pressure in the CFD analysis.

Fig. 7 also shows that the changes in the static pressure coefficient distribution which are induced by increasing the blockage ratio, vary considerably along the blade span. More specifically, when increasing the blockage ratio from 0.01 to 0.197 the percentage change in the minimum static pressure coefficient is lower at $r / R=0.94(4.5 \%)$ than at $r / R=0.6(7.1 \%)$. This is because the outboard blade sections are dominated by the rotational velocity component, so changes in the (effective) axial velocity incident on the blade (by increasing the blockage ratio) have a less significant effect on the static pressure distribution on the outboard blade sections than the inboard blade sections. Furthermore, changes in the suction can be loosely interpreted as equivalent to changes in the the angle of attack at a rate of approximately $1^{\circ}$ per 0.1 change in the minimum $C_{\text {pre }}$ (see Fig. 12). It can therefore be inferred from a combination of Fig. 12 and Fig. 7 that increasing the blockage ratio from 0.01 to 0.197 leads to an angle of attack increase of around $1^{\circ}$ at $r / R=0.94$. Hence even small changes in the static pressure distribution due to blockage should be expected to alter blade loads and the likelihood of cavitation inception significantly.

Comparing rotor designs, Fig 6 shows that at the same tip-speed-ratio and blockage ratio, Rotor 1 experiences a lower minimum suction pressure than Rotor 2. This is because Rotor 1 exerts less thrust on the flow (at the same blockage ratio and tip-speed-ratio), due to its shorter chord length and higher twist angle. With lower thrust applied to the flow, the axial velocity through the swept area of the rotor is higher for Rotor 1. By considering a velocity triangle, it can be inferred that (at the same blockage ratio and tip-speed-ratio) Rotor 1 therefore experiences a greater angle of attack and relative velocity magnitude than Rotor 2. Hence, Rotor 1 experiences a stronger suction peak at the same tip-speed-ratio and blockage ratio, as shown in Fig. 8. With a stronger suction peak, Rotor 1 will cavitate at a lower tip-speed-ratio than Rotor 2 (when operating at the same blockage ratio). Hence, more restrictive limitations will need to be applied on the operating conditions of Rotor 1 than Rotor 2, in order to avoid cavitation inception. 


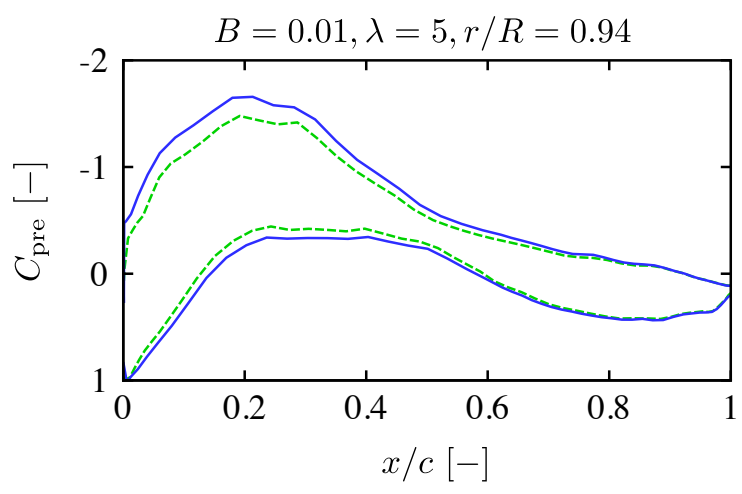

Rotor 1

Figure 8: Surface pressure coefficient in the chordwise direction on a slice through the blade of Rotor 1 and Rotor 2 (normal to the blade axis) at $r / R=0.94$. The blockage ratio $B=0.01$ and the tip-speed-ratio $\lambda=5$.

\subsection{Tip-speed-ratio and Submersion Depth Restrictions} either the maximum operational tip-speed-ratio can be limited or the minimum submersion depth can be limited. In order to specify such restrictions, Fig. 9 shows the minimum static pressure experienced by both rotor designs over the entire range of tip-speed-ratios, for two different submersion depths. Fig. 9 (a) shows the minimum static pressure experienced by both rotors with a tip submersion depth of $8 \mathrm{~m}$, while 9 (b) shows the minimum static pressure experienced by both rotors with the tip submersion depth reduced to $0 \mathrm{~m}$ (where the blades just skim the surface). A tip submersion depth of $8 \mathrm{~m}$ is likely to be typical for bed mounted devices, such as those installed at the MeyGen Ltd. (2014) site. However, some rotors may be installed below a floating support structure (such as the Scotrenewables SR250), with a much shallower submersion depth. To address this case, Fig. 9 (b) shows the minimum static pressure experienced by both rotors when the tip submersion depth is reduced to $0 \mathrm{~m}$. This submersion depth was specifically chosen to investigate the limit of minimum submersion depth, when the rotors are most likely to cavitate. As the hydrostatic pressure is applied during the post-processing stage (and is linear), other intermediate submersion depths could also be considered by translating the curves in Fig. 9 in the vertical direction. However, these intermediate depths are not shown here for brevity.

At a tip submersion depth of $8 \mathrm{~m}$, neither rotor is likely to cavitate over the range of tip-speed-ratios considered because the minimum static pressure head is greater than the vapour pressure head of seawater $(0.24 \mathrm{~m})$. However, with a tip submersion depth of $0 \mathrm{~m}$, cavitation inception is likely to occur if the minimum static pressure drops below the horizontal dashed line in Fig. 9 (b) (which corresponds with the vapour pressure head of seawater). Furthermore, if the submersion depth is fixed (perhaps due to the nature of the support structure), then the intersection between the computed curves and the horizontal dashed line in Fig. 9 (b) represents the maximum permissible tip-speed-ratio to avoid cavitation inception.

Traversing to the left along the horizontal dashed line in Fig. 9 (b), shows that increasing the blockage ratio from 0.01 to 0.197 reduces the maximum permissible tip-speed-ratio by 0.24 for Rotor 1 and by 0.27 for Rotor 2. This is because the strength of the suction peak increases with blockage ratio (see Fig. 6) and hence the tip-speed-ratio of the rotor has to reduce in order to limit the strength of the suction peak and avoid cavitation. Alternatively, if the submersion depth is not fixed and it is desirable to maintain the same maximum operational tip-speed-ratio, then the rotor submersion depth would have to increase by $0.53 \mathrm{~m}$ (traversing downwards across blockage curves at fixed $\lambda$ ) to maintain the same minimum static pressure as the blockage ratio is increased from 0.01 to 0.197 (at a tip-speed-ratio of 6 ).

Comparing rotor designs, Fig. 9 (b) shows that at a blockage ratio of 0.197 the maximum permissible tip-speed-ratio for Rotor 2 is 0.48 higher than Rotor 1 . This is because Rotor 2 experiences a weaker suction peak than Rotor 1 at the same tip-speed-ratio (see Fig. 6) and hence can be operated at a higher tip-speed- 
(a)

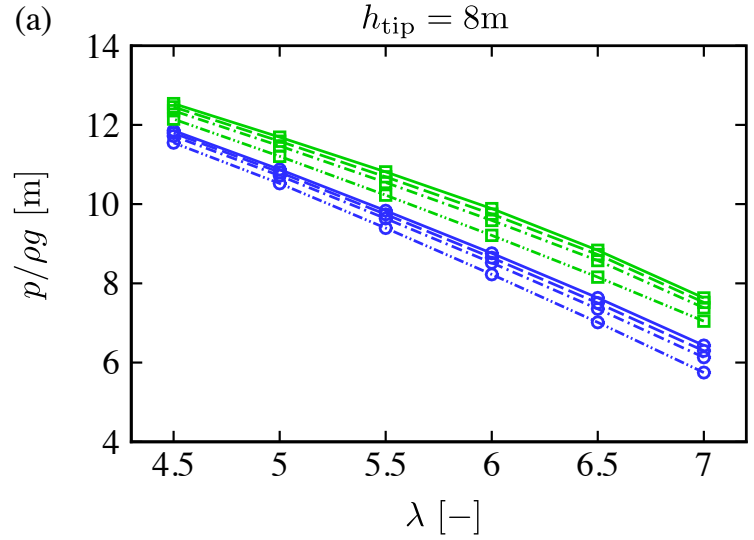

$\multimap$ Rotor $1, B=0.01-\bullet-$ Rotor $1, B=0.065$

$\square$ Rotor $2, B=0.01-\square-$ Rotor $2, B=0.065$ (b)

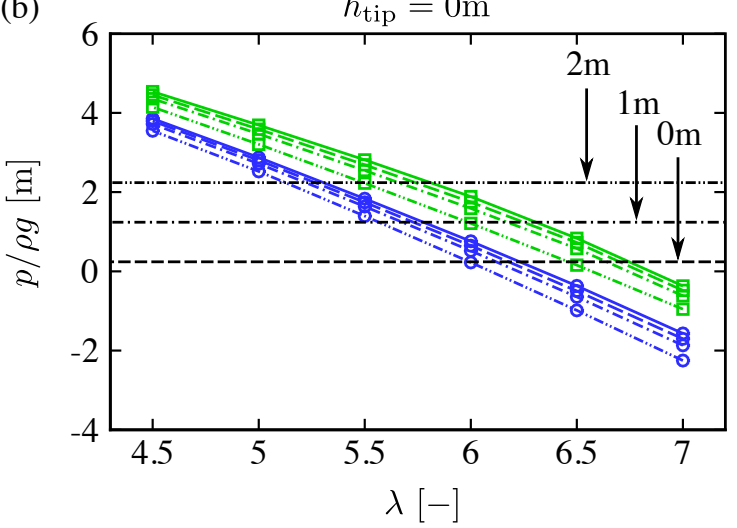

Figure 9: Minimum static pressure head on the blade suction surface for tip submersion depths of (a) $8 \mathrm{~m}$ and (b) 0m. The horizontal dashed, dashed dotted and dashed double dotted lines indicate safety margins of $0 \mathrm{~m}$, $1 \mathrm{~m}$ and $2 \mathrm{~m}$ respectively, relative to the vapour pressure head of seawater $(0.24 \mathrm{~m})$.

ratio before cavitation inception is likely to occur. Alternatively, if the submersion depth is not fixed, then the submersion depth of Rotor 1 would have to be increased by $0.98 \mathrm{~m}$ to attain the same minimum static pressure as Rotor 2 at a tip-speed-ratio of 6.0 .

\subsection{Hydrodynamic Safety Margin}

Ambient turbulence, free surface waves and velocity shear lead to unsteady pressure fluctuations that have not been modelled in the computations carried out in this paper. These unsteady fluctuations may result in cavitation inception, even if the minimum static pressure under steady conditions is greater than the vapour pressure of seawater. For example, the highly sheared velocity profile (that is present at many tidal energy sites) leads to an increase in the axial velocity (and hence angle of attack) near the top of the water column. As the angle of attack increases, the strength of the suction peak increases, which pushes the blade closer to cavitation than it would be in uniform flow. Furthermore, the presence of free surface waves leads to a transient submersion depth, which locally reduces the hydrostatic pressure contribution (during wave troughs) and pushes the rotor closer to cavitation.

As these unsteady fluctuations have not been modelled directly, a hydrodynamic safety margin is required. For consistency with the preceding analysis, this safety margin will be specified as an increase in the minimum allowable static pressure head under steady flow conditions. This additional static pressure head is required to prevent the fluid from cavitating as a result of the unsteady static pressure fluctuations arising from velocity shear, surface waves, ambient turbulence and the transient submersion depth induced by surface waves. Rather than suggesting an appropriate value, the effect of specifying such a safety margin on the range of operating conditions that can be achieved by the rotor will be demonstrated instead. This approach was taken because appropriate safety margins are likely to be dependent on the local environmental conditions (they are site specific). An additional safety margin may also be required for other effects such as marine fowling, but these effects have not been considered here.

For bed mounted devices, where the tip submersion depth is large, the minimum static pressure may already be sufficiently large that an additional static pressure head is not necessary. For example, even if the minimum static pressure head is reduced by $5 \mathrm{~m}$ in Fig. 9 (a), both Rotor 1 and Rotor 2 are not likely to cavitate over the entire range of operating conditions. Hence, if the required safety margin is less than $5 \mathrm{~m}$, then no restriction on the rotor operating conditions is required at a tip submersion depth of $8 \mathrm{~m}$.

However, for devices operating higher up in the water column, where the tip submersion depth is small, an increase in the minimum allowable static pressure head (by including a safety margin) is essential to prevent the device from cavitating. In Fig. 9 (b) the horizontal dashed dotted and horizontal dashed double 
dotted lines indicate additional static pressure heads of $1 \mathrm{~m}$ and $2 \mathrm{~m}$ respectively, that could be selected as an appropriate safety margin (depending on the local environmental conditions). At this submersion depth, the chosen safety margin will significantly impact the maximum permissible tip-speed-ratio that can be achieved by the rotor (if it is not possible to increase the submersion depth). For example, applying a safety margin of $1 \mathrm{~m}$ (pressure head) to Rotor 1 at $B=0.197$ reduces the maximum permissible tip-speed-ratio by 0.42 (a $7.0 \%$ reduction in rotational speed). As discussed in Section 5.3, this reduction in maximum permissible tip-speed-ratio has significant consequences for the thrust and power coefficients that can be achieved by the rotor.

However, if it is possible to increase the submersion depth of the rotor (rather than limiting the tipspeed-ratio), Fig. 9 (b) can also be used to select an appropriate submersion depth for the rotor that does not restrict the range of operational tip-speed-ratios and includes the required safety margin. For example, to maintain a safety margin of $2 \mathrm{~m}$ and achieve a tip-speed-ratio of 7 at a blockage ratio of 0.197 , Rotor 1 requires a minimum submersion depth of $4.5 \mathrm{~m}(2.0 \mathrm{~m}+0.25 \mathrm{~m}--2.25 \mathrm{~m})$, while Rotor 2 requires a minimum submersion depth of only $3.2 \mathrm{~m}(2.0 \mathrm{~m}+0.25 \mathrm{~m}--0.95 \mathrm{~m})$. However, it may not always be desirable to increase the submersion depth in preference to limiting the range of operational tip-speed-ratios. This is because the velocity profile at some tidal energy sites is highly sheared, with a greater proportion of the incident kinetic energy flux residing near the top of the water column. Hence, reducing the submersion depth at these sites results in a lower potential for energy extraction, despite not having to limit the tip-speed-ratio to avoid cavitation inception.

\subsection{Rotor Performance}

For rotors which cannot increase their submersion depth, the tip-speed-ratio restriction may significantly reduce the maximum thrust and power coefficients that can be achieved. Fig. 10 shows the power and thrust coefficients $\left(C_{P}\right.$ and $C_{T}$ respectively) for Rotor 1 and Rotor 2, which have been defined as:

$$
\begin{aligned}
C_{T} & =\frac{T}{\frac{1}{2} \rho U_{\infty}^{2} A} \\
C_{P} & =\frac{P}{\frac{1}{2} \rho U_{\infty}^{3} A}
\end{aligned}
$$

where $T$ is the rotor thrust, $P$ is the rotor power, $\rho=1025 \mathrm{~kg} / \mathrm{m}^{3}$ is the fluid density and $A$ is the swept area of the rotor. To assist in the ensuing analysis, a polynomial curve fit has been used to estimate the maximum power coefficient in each domain and the approximate tip-speed-ratio that it occurred at. The maximum power coefficients are also shown in Fig. 10 and are connected by a black dashed line.

Fig. 10 shows that increasing the blockage ratio at constant tip-speed-ratio (following the solid black line) leads to an increase in device thrust and power coefficients (for both rotor designs). Nishino and Willden (2012) have attributed this increase in thrust and power at higher blockage ratios to the acceleration of the bypass flow (the flow which does not pass through the device swept area). As the bypass flow accelerates due to the downstream flow passage constriction, a greater static pressure drop develops in the bypass flow. The bypass flow static pressure must equalise with the core flow static pressure, far upstream and downstream of the device. Hence, a greater static pressure drop is able to build up across the core flow passage, which is balanced by increases device thrust. The device is therefore able to extract more power from the flow, by achieving a greater static pressure head drop across the core flow passage.

However, operating at a constant tip-speed-ratio while increasing the blockage ratio does not lead to the maximum power coefficient that is available in the higher blockage domain. To achieve the maximum power coefficient, the rotor must follow the approximate traverse indicated by the black dashed line in Fig. 10. For Rotor 1 , this requires a higher tip-speed-ratio in the higher blockage domain, while a lower tip-speed-ratio is required for Rotor 2 in the lower blockage domain.

To explain the required change in tip-speed-ratio with blockage ratio, Fig. 11 shows the power coefficient variation with the mass flow rate coefficient $\left(C_{\dot{m}}\right)$. Here the mass flow rate coefficient is defined as the ratio of the mass flux through the swept area of the rotor to the mass flux through the swept area of the 
(a)

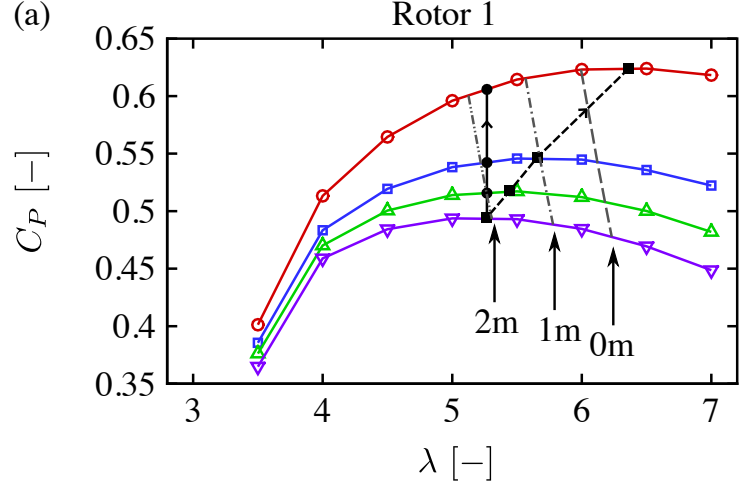

(c)

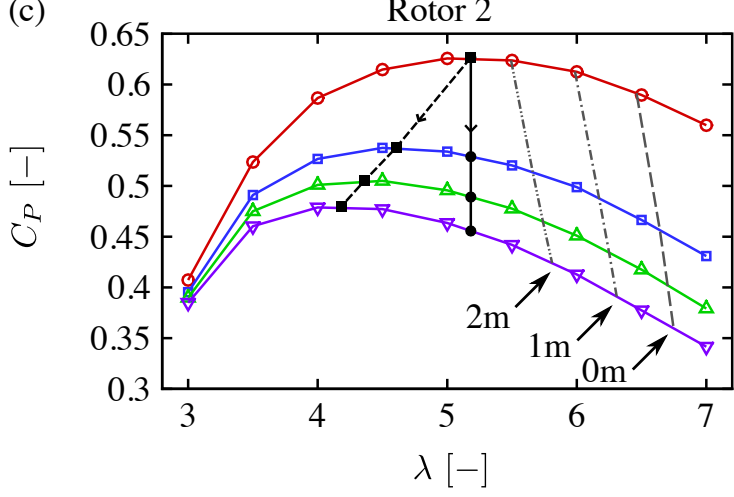

$\nabla-B=0.01$

$\triangle B=0.065$ (b)

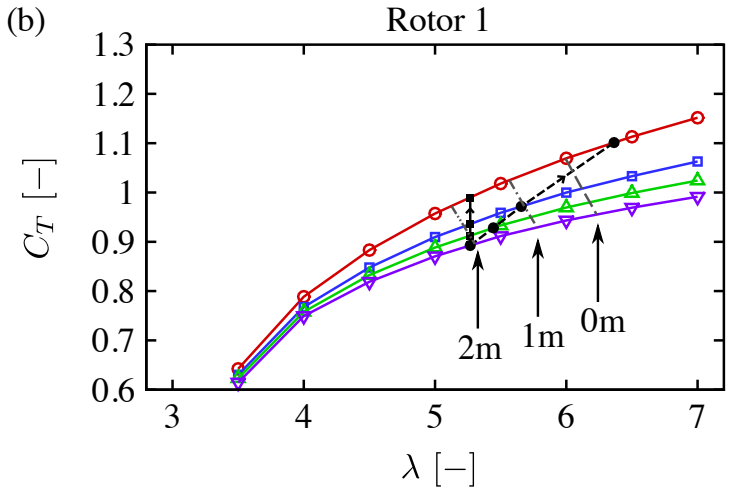

(d)

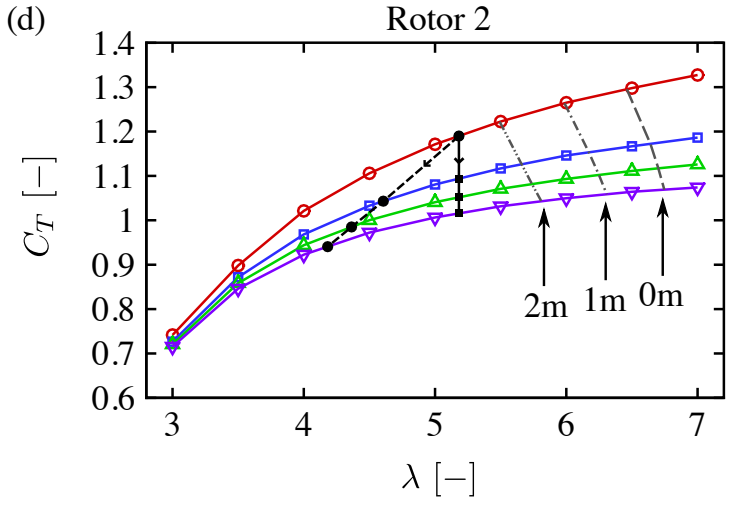

Figure 10: Power and thrust coefficients for Rotor 1 and Rotor 2 over a range of blockage ratios and tip-speed-ratios. The black solid lines indicate constant tip-speed-ratio operation, while the black dashed lines indicate the approximate trajectory required to achieve the maximum power coefficient at each blockage ratio. The grey lines indicate the maximum permissible tip-speed-ratios for a device with a tip submersion depth of $0 \mathrm{~m}$ and safety margins of $0 \mathrm{~m}, 1 \mathrm{~m}$ and $2 \mathrm{~m}$.

rotor with no device present. For Rotor 1, increasing the blockage ratio (from 0.01 to 0.197) at constant tip-speed-ratio leads to a mass flow rate coefficient through the rotor plane that is too high to maximise the power coefficient. This is because the rotor provides insufficient thrust (due to its low solidity) to divert the flow into the bypass flow passage. Hence, the rotor must spin faster (increase its tip-speed-ratio) in order to provide the additional thrust required (see Fig. 10 (b) and (d)) to divert the flow into the bypass flow passage and decrease the mass flow rate coefficient as blockage is increased.

However, if the maximum permissible tip-speed-ratio is limited (due to the cavitation restrictions discussed in Section 5.2), it may not be possible to maximise the power coefficient by increasing the tipspeed-ratio. For example, the grey solid, dashed and dashed dotted lines in Fig. 10 indicate the maximum permissible tip-speed-ratios for Rotor 1 and Rotor 2 with safety margins of $0 \mathrm{~m}, 1 \mathrm{~m}$, and $2 \mathrm{~m}$ (respectively) applied to a submersion depth of $0 \mathrm{~m}$. These lines directly correspond with the horizontal safety margin lines in Fig. 9 (b). In order to avoid the onset of cavitation, operating conditions to the right of these lines are not permitted.

The performance of Rotor 2 will not be affected by a safety margin of $2 \mathrm{~m}$ or less, since the maximum power coefficient that is available at each blockage ratio occurs at a tip-speed-ratio that is lower than the maximum permissible tip-speed-ratio. This is because Rotor 2 has higher solidity and therefore does not need as high a tip-speed-ratio as Rotor 1 to provide the necessary thrust to maximise the power coefficient in each domain. Conversely, Rotor 1 may not be able to achieve the maximum power coefficient that is available in each domain, due to the required safety margin. In the high blockage domain $(B=0.197)$ for example, Rotor 1 can achieve a maximum power coefficient of 0.624 at a tip-speed-ratio of 6.36 . However, 

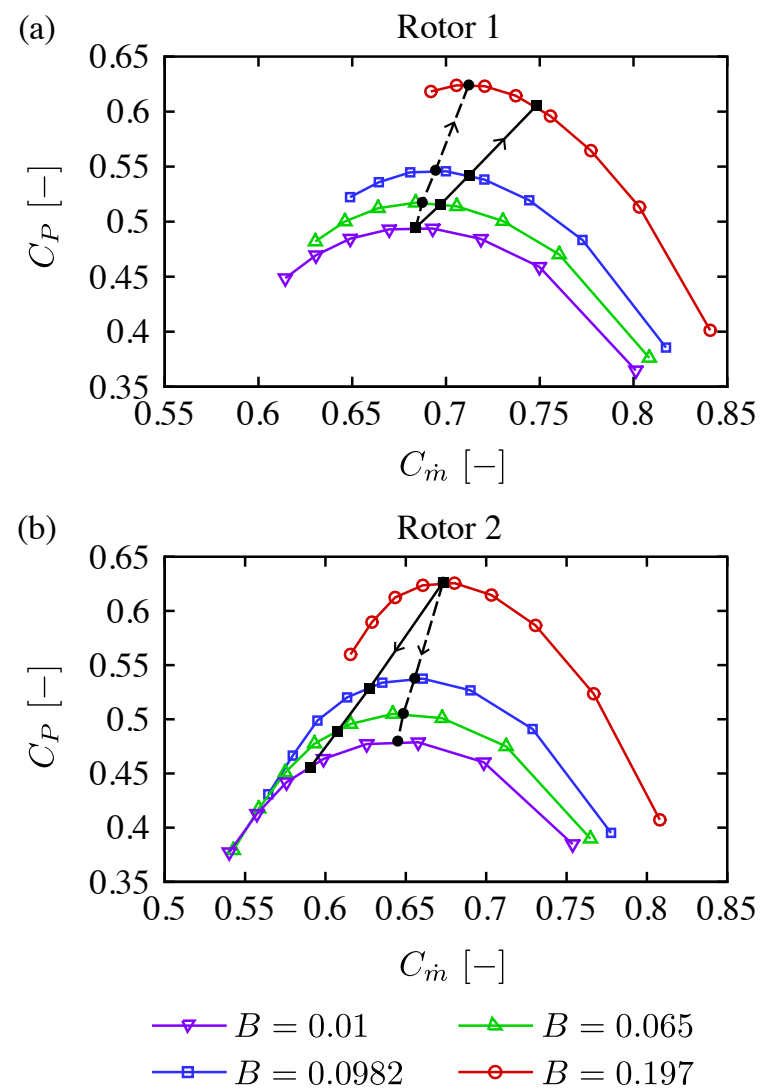

Figure 11: Power coefficient variation with mass flow rate coefficient for Rotor 1 and Rotor 2 over a range of blockage ratios and tip-speed-ratios. The black solid line indicates constant tip-speed-ratio operation, while the black dashed line indicates the approximate trajectory required to maximise the power coefficient at each blockage ratio.

with a safety margin of $2 \mathrm{~m}$, this tip-speed-ratio is beyond the maximum permissible tip-speed-ratio and hence the tip-speed-ratio is limited to 5.13 instead. With this tip-speed-ratio restriction, the maximum power coefficient of the rotor is limited to 0.601 (a performance decrement of 0.023 or 3.69\%). At high blockage ratios, it follows that due to the cavitation restriction on the rotor tip-speed-ratio, Rotor 2 (the high thrust rotor) will always outperform Rotor 1, unless Rotor 1 is sufficiently submerged that the tipspeed-ratio restriction is not necessary (but even then it can only approach the performance of Rotor 2 which is specifically designed for higher blockage). This is because Rotor 2 achieves its maximum power coefficient at a lower tip-speed-ratio than Rotor 1 and the cavitation restriction itself occurs at a higher tip speed for Rotor 2 (due to its weaker suction peak).

\section{Cavitation Analysis using the BE-AD method}

Having carried out a cavitation analysis using the results of the blade resolved computations, a separate cavitation analysis was carried out using the results of the BE-AD computations. To carry out this analysis, the angle of attack $(\alpha)$ and relative velocity magnitude $\left(U_{\text {rel }}\right)$ were extracted from the simulated flow field and used to compute the spanwise distribution of the minimum static pressure (with the blade at top dead centre) using equation 5.

$$
p=p_{\text {Atm }}+\rho g\left(h_{\text {tip }}+R-r\right)+C_{\text {pre }}(\alpha(r))\left[\frac{1}{2} \rho U_{\text {rel }}^{2}(r)\right]
$$




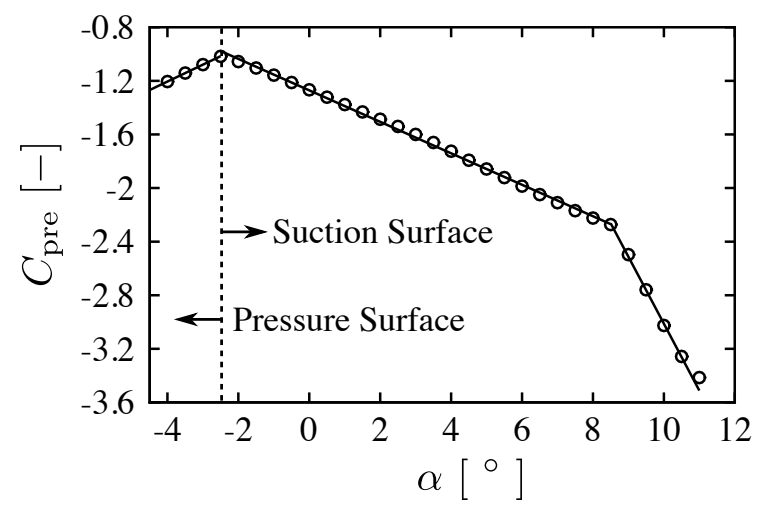

- Computed L Linear Curve Fit

Figure 12: Minimum static pressure coefficient (as defined in equation 2 with $\Omega=0$ ) on the surface of the RIS $\varnothing$-A1-24 aerofoil at a chord based Reynolds number of 12 million.

The minimum static pressure coefficient $\left(C_{\text {pre }}(\alpha)\right)$ was then extracted from a set of $2 \mathrm{D}$ aerofoil computations of the RIS $\varnothing-A 1-24$ aerofoil over a range of angles of attack, as shown in Fig. 12. This is the standard approach for carrying out blade element based cavitation analyses.

While the RANS solver in the BE-AD method can capture spanwise flow (which is mainly generated by the nacelle as the flow is deflected radially outwards), the Blade Element (BE) aspect of the BE-AD method does not account for spanwise flow effects. This is because the forces acting on each aerofoil section are assumed to only be due to the velocity components normal to the aerofoil section. Hence, even if a significant spanwise flow component is computed by the RANS solver, it is not accounted for in the calculation of the angle of attack, relative velocity magnitude and resultant pressure for the cavitation analysis.

(a)

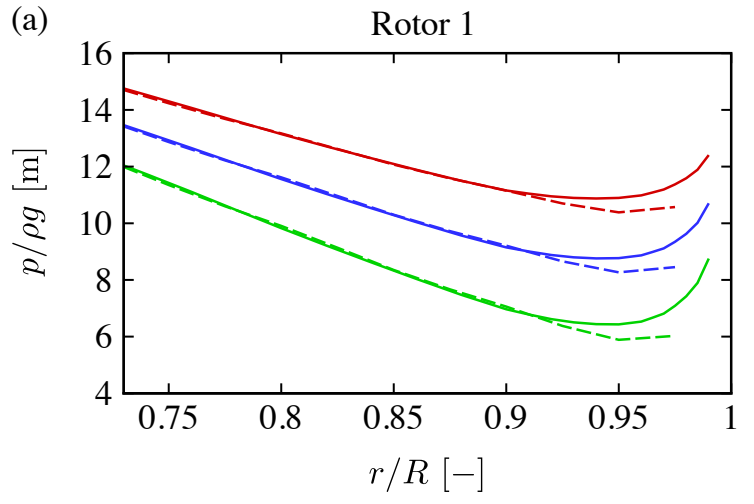

Blade Resolved, $\lambda=5$

$\mathrm{BE}-\mathrm{AD}, \lambda=5$ (b)
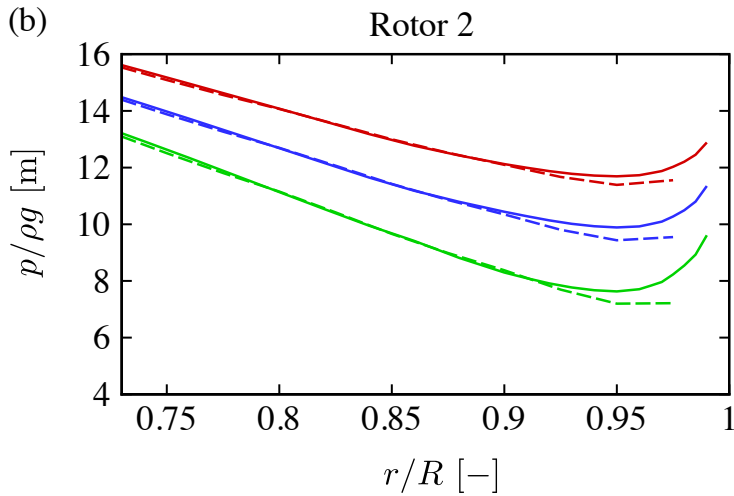

Blade Resolved, $\lambda=7$

$\mathrm{BE}-\mathrm{AD}, \lambda=7$

Figure 13: Spanwise variation of the minimum static pressure acting on each aerofoil section, expressed in terms of a pressure head.

BE based methods can indirectly account for some aspects of the effect of spanwise flow on the outboard blade sections by applying a Glauert correction factor to the axial induction factor. The increase in the axial induction factor reduces the angle of attack, which implicitly reduces the strength of the suction peak on the outboard blade sections (see Fig. 12). As shown in Fig. 13, the BE-AD method therefore predicts that the minimum static pressure does not occur at the very tip of the blade but slightly inboard at $r / R=0.95$ (and remains approximately at this location with changes in tip-speed-ratio). While the spanwise location 
of the minimum static pressure is in good agreement with the blade resolved computations, the magnitude is considerably different. This is because the Glauert correction is only an approximation and does not sufficiently account for spanwise flow effects on the outboard sections of real rotors. Similar behaviour is likely to be exhibited by any BE based methods that only use a Glauert correction to account for spanwise flow effects on the outboard blade sections.

(a)

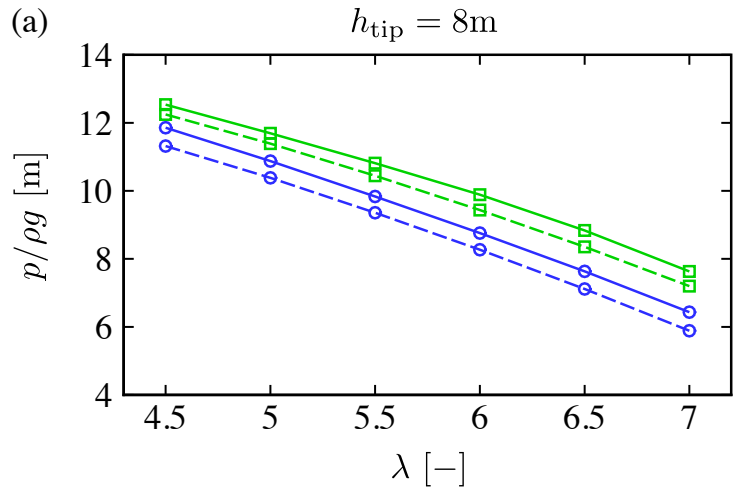

- Rotor 1, Blade Resolved (b)

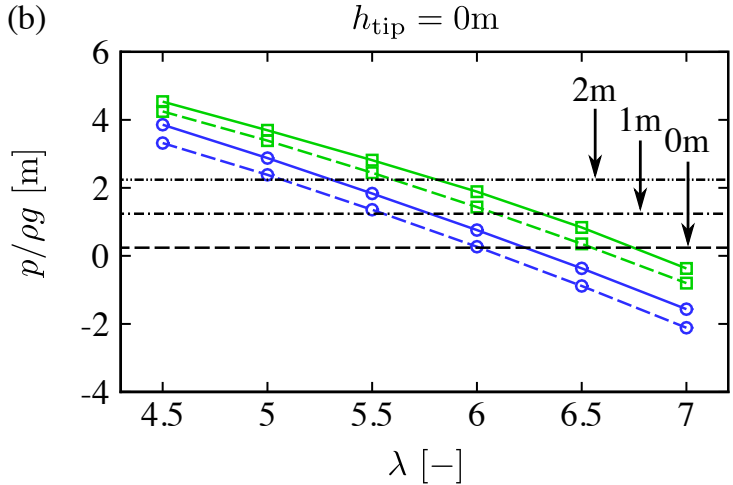

$\square$ Rotor 2, Blade Resolved

-- -- Rotor 2, BE-AD

Figure 14: Minimum static pressure head on the blade suction surface for tip submersion depths of (a) 8m and (b) 0m. The horizontal dashed, dashed dotted and dashed double dotted lines indicate safety margins of $0 \mathrm{~m}, 1 \mathrm{~m}$ and $2 \mathrm{~m}$ respectively, relative to the vapour pressure head of seawater $(0.24 \mathrm{~m})$.

Fig. 14 compares the magnitude of the minimum static pressure computed using the BE-AD and blade resolved approaches. At the same tip-speed-ratio, the BE-AD approach predicts a minimum static pressure head that is approximately $0.4 \mathrm{~m}$ lower than the blade resolved approach. Alternatively, for the same minimum static pressure head, the BE-AD approach predicts a maximum permissible tip-speed-ratio that is 0.25 lower than the blade resolved approach. Hence, rotors which are currently designed using Blade Element based algorithms are overly conservative, with a greater effective safety margin than may have originally been intended. It may therefore be possible to either install rotors (that were designed using the $\mathrm{BE}-\mathrm{AD}$ approach) higher up in the water column or extend their range of operational tip-speed-ratios.

The reader may also note that the minimum static pressure in Fig. 14 does not form a traditional cavitation 'bucket' shape, where the minimum static pressure increases at high tip-speed-ratios (Carlton, 1994). This is because the angle of attack was sufficiently high in the computations carried out in this work that the minimum static pressure occurred on the suction surface of the blade over all the considered tipspeed-ratios. However, at higher tip-speed-ratios (not considered in this work), the angle of attack reduces and the minimum static pressure will eventually transition to the pressure surface of the blade (see Fig. 12) and will start to increase (forming a bucket shape). This type of cavitation may occur with turbines using overspeed control, unless they are carefully designed.

\section{Cavitation Number}

An alternative (but equivalent) approach to presenting the minimum static pressure on the blade surface is to define a cavitation number $\sigma$ (Carlton, 1994),

$$
\sigma=\frac{p-p_{\mathrm{v}}}{\frac{1}{2} \rho\left[U_{\infty}^{2}+(\Omega R)^{2}\right]}
$$

where $p_{\mathrm{v}}$ is the vapour pressure of seawater $(2.4 \mathrm{kPa})$ and $p$ is the minimum static pressure on the blade surface (defined using equation 1 for the blade resolved computations and equation 5 for the BE-AD computations). With this definition of the cavitation number, cavitation inception may only occur if $\sigma<0$. 
(a)

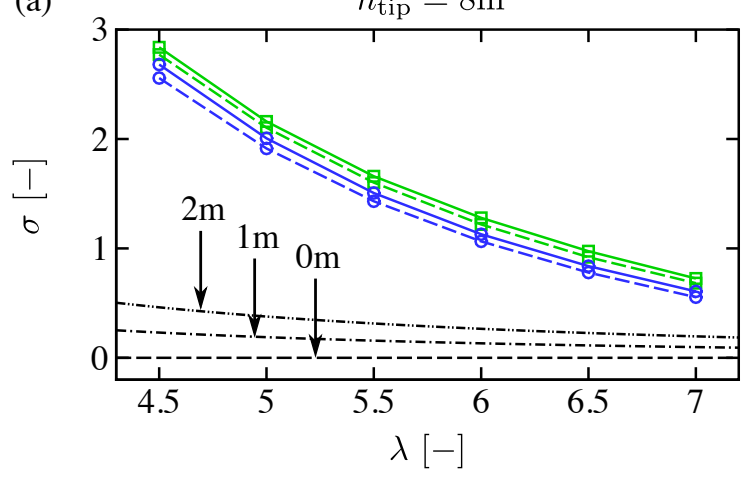

- Rotor 1 Blade Resolved (b)

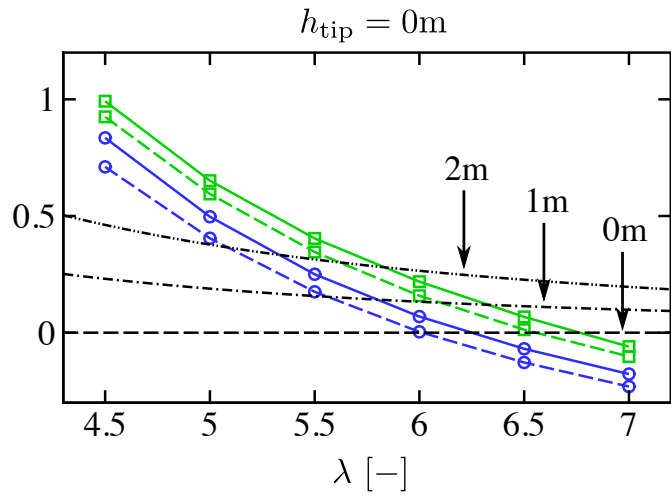

Rotor 2 Blade Resolved

--•-- Rotor 2 BE-AD

Figure 15: Cavitation number for tip submersion depths of (a) $8 \mathrm{~m}$ and (b) 0m. The dashed, dashed dotted and dashed double dotted lines indicate safety margins of $0 \mathrm{~m}, 1 \mathrm{~m}$ and $2 \mathrm{~m}$ respectively. increase in the strength of the suction peak of each device in the array could be conservatively accounted for by the additional global safety margin. Furthermore, the additional safety margin can be computed by simulating a single rotor with a blockage ratio that corresponds with the highest local blockage ratio in the array (using the approach carried out in this work). For example, for an array of devices with diameter blockage that may be experienced in the array is 0.197 . Hence, if Rotor 2 were used for all the devices in this array, an additional safety margin of $0.53 \mathrm{~m}$ would conservatively account for the (unknown) increase in the strength of the suction peak of all devices in the array and thus avoid the occurrence of cavitation conditions. 


\section{Conclusions}

Tidal turbines are likely to operate in close proximity with other devices, utilising the increased blockage provided by the sea bed, free surface and other neighbouring devices to increase the maximum power coefficient that can be extracted by the devices. However, current cavitation analyses do not account for the increased strength of the blade suction peak at non-negligible blockage ratios. In this work, a cavitation analysis was performed using a series of blade resolved computations to capture the increased suction peak strength at higher blockage ratios. Our analysis suggests that increasing the blockage ratio from 0.01 to 0.197 reduces the minimum static pressure head in the fluid by approximately $0.5 \mathrm{~m}$ for our rotor design (which is representative of some full-scale devices). Hence, it may be necessary to either increase the submersion depth of the rotor by $0.5 \mathrm{~m}$ or reduce the maximum permissible tip-speed-ratio, in order to mitigate the reduction in minimum static pressure at a blockage ratio of 0.197 .

It is essential to include a hydrodynamic safety margin in cavitation analyses to account for the unsteady pressure fluctuations induced by the sheared velocity profile, free surface waves and ambient turbulence. In this work, a safety margin was introduced in the form of an increase in the minimum allowable static pressure head. While the magnitude of the safety margin adopted will depend on the local environmental conditions, the effect of increasing the safety margin by $1 \mathrm{~m}$ and $2 \mathrm{~m}$ was investigated in this work. Our analysis suggests that increasing the safety margin by $1 \mathrm{~m}$ could be achieved by either increasing the submersion depth by $1 \mathrm{~m}$ or reducing the maximum permissible tip-speed-ratio of our rotor by approximately 0.4 (if the submersion depth of the device is fixed). Furthermore, it has been shown that the cavitation restriction on tip-speed-ratio may restrict the power coefficient that real rotors can achieve as device blockage is increased. Therefore, to achieve the optimum power coefficient it is necessary to design rotors specifically for their intended blockage ratio and tip-speed-ratio.

The majority of tidal turbines are currently designed using Blade Element based approaches. Despite the inclusion of a Glauert correction, these approaches do not adequately account for spanwise flow effects, since the Glauert correction factor is only an approximation for real rotors. When carrying out a cavitation analysis, accurate representation of spanwise flow effects is essential since spanwise flow effects reduce the strength of the suction peak on the outboard blade sections. As the strength of the suction peak reduces, the minimum static pressure in the fluid increases, delaying the onset of cavitation. In this work, BE-AD computations were carried out and compared with the results of blade resolved computations, to identify and quantify the discrepancies in the cavitation analysis induced by inaccurate representation of spanwise flow effects. Our analysis suggests that cavitation analyses based on Blade Element type approaches are currently overly-conservative, with a safety margin that is larger than intended. Furthermore, for the rotors analysed in this work it would be possible to either increase the range of operational tip-speed-ratios by 0.25 or reduce the submersion depth by $0.4 \mathrm{~m}$ (beyond those envisaged by BE-AD analysis) while maintaining the same safety margin.

\section{Acknowledgement}

The authors would like to thank EPSRC and Uniper Technologies Ltd. for funding the CASE-Studentship for this project and the Advanced Research Computing facility at the University of Oxford where the computations were performed.

\section{References}

Adcock, T. A. A., Draper, S., Nishino, T., 2015. Tidal power generation - a review of hydrodynamic modelling. Proc IMechE Part A: J Power and Energy 229 (7), 755771.

Bahaj, A. S., Molland, A. F., Chaplin, J. R., Batten, W. M. J., 2007. Power and thrust measurements of marine current turbines under various hydrodynamic flow conditions in a cavitation tunnel and a towing tank. Renewable Energy 32, 407-426.

Batten, W., Bahaj, A., Molland, A., 2008. The prediction of the hydrodynamic performance of marine current turbines. Renewable Energy 33 (5), 1085-1096.

Belloni, C. S. K., 2013. Hydrodynamics of Ducted and Open-Centre Tidal Turbines. DPhil Thesis, University of Oxford.

Burton, T., Jenkins, N., Sharpe, D., Bossanyi, E., 2011. Wind Energy Handbook. John Wiley \& Sons Ltd., West Sussex, UK. 
Carlton, J. S., 1994. Marine Propellers and Propulsion. Butterworth-Heinemann, Burlington, USA.

Consul, C., Willden, R. H. J., McIntosh, S. C., 2013. Blockage effects on the hydrodynamic performance of a marine cross flow turbine. Phil. Trans. R. Soc. A. 371.

DNV GL, October 2015. Tidal Turbines - Rules and Standards. Tech. Rep. DNVGL-ST-0164.

Gant, S., Stallard, T., July 2008. Modelling a tidal turbine in unsteady flow. In: International Offshore and Polar Engineering Conference. Vancouver, Canada, pp. 473-479.

Glauert, H., 1935. Airplane propellers. In: Aerodynamic Theory. Julius Springer, Berlin, pp. 169-360.

510 Goundar, J., Ahmed, M., 2013. Design of a horizontal axis tidal current turbine. Applied Energy 111, 161-174.

Grogan, D., Leen, S., Kennedy, C., Brádaigh, C. O., 2013. Design of composite tidal turbine blades. Renewable Energy 57, $151-162$.

Luo, J. Y., Issa, R. I., Gosman, A. D., 1994. Prediction of impeller-induced flows in mixing vessels using multiple frames of reference. In: IChemE Symposium Series. 136. pp. 549-556.

515 McIntosh, S., Fleming, C., Willden, R., 2011. Embedded RANS-BEM tidal turbine design. In: Ninth European Wave and Tidal Energy Conference. Southampton UK.

Menter, F. R., 1994. Two-equation eddy-viscosity turbulence models for engineering applications. AIAA Journal 32(8), 15981605.

MeyGen Ltd., 2014. Decommissioning programme. Tech. Rep. MEY-10-70-HSE-002-D-.

520 Ning, A., Dykes, K., 2014. Understanding the benefits and limitations of increasing maximum rotor tip speed for utility scale wind turbines. Journal of Physics: Conference Series 524, 012087.

Nishino, T., Willden, R. H. J., 2012. Effects of 3-D channel blockage and turbulent wake mixing on the limit of power extraction by tidal turbines. International Journal of Heat and Fluid Flow 37, 123-135.

Schluntz, J., Willden, R. H. J., 2015. The effect of blockage on tidal turbine rotor design and performance. Renewable Energy $81,432-441$.

Vennel, R., Funke, S., Draper, S., Stevens, C., Divett, T., 2015. Designing large arrays of tidal turbines: a synthesis and review. Renewable and Sustainable Energy Reviews 41, 454-472.

Wang, D., Atlar, M., Sampson, R., 2007. An experimental investigation on cavitation, noise, and slipstream characteristics of ocean stream turbines. Proceedings of the Institution of Mechanical Engineers, Part A: Journal of Power and Energy 221 (2), 219-231.

Wimshurst, A., Willden, R. H. J., October 2016. Computational analysis of blockage designed tidal turbine rotors. In: Proceedings of the 2nd International Conference on Renewable Energies Offshore (RENEW 2016). Lisbon, Portugal, pp. 587-597.

Wimshurst, A., Willden, R. H. J., September 2017. Spanwise flow corrections for tidal turbines. In: 12th European Wave and Tidal Energy Conference (EWTEC 2017). Cork, Ireland. 